\title{
Layer-Wise De-Training and Re-Training for ConvS2S Machine Translation
}

\author{
HONGFEI YU, XIAOQING ZHOU, XIANGYU DUAN, and MIN ZHANG, Soochow University
}

\begin{abstract}
The convolutional sequence-to-sequence (ConvS2S) machine translation system is one of the typical neural machine translation (NMT) systems. Training the ConvS2S model tends to get stuck in a local optimum in our pre-studies. To overcome this inferior behavior, we propose to de-train a trained ConvS2S model in a mild way and retrain to find a better solution globally. In particular, the trained parameters of one layer of the NMT network are abandoned by re-initialization while other layers' parameters are kept at the same time to kick off re-optimization from a new start point and safeguard the new start point not too far from the previous optimum. This procedure is executed layer by layer until all layers of the ConvS2S model are explored. Experiments show that when compared to various measures for escaping from the local optimum, including initialization with random seeds, adding perturbations to the baseline parameters, and continuing training (con-training) with the baseline models, our method consistently improves the ConvS2S translation quality across various language pairs and achieves better performance.
\end{abstract}

\section{CCS Concepts: • Computing methodologies $\rightarrow$ Machine translation;}

Additional Key Words and Phrases: ConvS2S, neural machine translation, local optimum

\section{ACM Reference format:}

Hongfei Yu, Xiaoqing Zhou, Xiangyu Duan, and Min Zhang. 2019. Layer-Wise De-Training and Re-Training for ConvS2S Machine Translation. ACM Trans. Asian Low-Resour. Lang. Inf. Process. 19, 2, Article 26 (November 2019), 15 pages.

https://doi.org/10.1145/3358414

\section{INTRODUCTION}

Neural machine translation (NMT) advanced state-of-the-art machine translation performance recently when compared to the traditional statistical machine translation (SMT) system. NMT mostly adopts an encoder-decoder framework that encodes a source sentence with certain neural information and decodes such information into the corresponding target sentence. The bridge between the encoder and the decoder is often modeled by the attention mechanism that summarizes the source-side relevant information when translating a target word.

Recurrent neural network (RNN)-based NMT is the seminal architecture that totally departs from traditional SMT architectures $[1,13,21]$. The following research explored alternative

This work was supported by the National Key R\&D Program of China (grant 2016YFE0132100) and the National Natural Science Foundation of China (grants 61673289 and 61273319).

Authors' addresses: H. Yu, X. Zhou, X. Duan (corresponding author), and M. Zhang, Soochow University; emails: \{hfyutravel, zxqchat\}@gmail.com, \{xiangyuduan, minzhang\}@suda.edu.cn.

Permission to make digital or hard copies of all or part of this work for personal or classroom use is granted without fee provided that copies are not made or distributed for profit or commercial advantage and that copies bear this notice and the full citation on the first page. Copyrights for components of this work owned by others than ACM must be honored. Abstracting with credit is permitted. To copy otherwise, or republish, to post on servers or to redistribute to lists, requires prior specific permission and/or a fee. Request permissions from permissions@acm.org.

(C) 2019 Association for Computing Machinery.

2375-4699/2019/11-ART26 \$15.00

https://doi.org/10.1145/3358414 
architectures that are non-RNN based and achieved comparable or better performance to the RNNbased NMT [7, 22]. The convolutional sequence-to-sequence (ConvS2S) machine translation system is one of such explorations. ConvS2S stacks deep convolution layers over both sides with the ability of capturing local adjacent features beneficial for target word generation [7]. Since the convolution is computed position independently, ConvS2S can be highly parallelized and runs much faster than RNN-based architecture.

Although ConvS2S attains good results over several language pairs, it is hard to be fully trained due to its deep layers of vast parameters. We observe that training ConvS2S often sticks to a local optimum, which is a common phenomenon in high-dimensional neural networks. In our prestudies of trying to escape from the local optimum, three kinds of measures are applied: initialization with random seeds, performing noise perturbation to the baseline parameters, and continuing training (con-training) with the baseline model. The investigation shows that all three measures cannot find solutions better than the baseline model.

To address this problem, we propose a de-training and re-training procedure to find a better solution globally. In particular, at each step of our procedure, one layer of ConvS2S is de-trained by re-initialization while other layers remain the same, followed by a re-training step based on the re-initialized layer and the untouched layers in the last step. These two steps repeat layer wisely until all layers of ConvS2S are explored. The whole procedure is executed to progressively escape from the local optimum.

Experimental results on large-scale Chinese-to-English and German-to-English machine translation tasks show that our training procedure for ConvS2S is able to find better solutions than the baseline ConvS2S model, resulting in significant improvements of 2.964 BLEU points on the Chinese-to-English task and 0.94 BLEU points on the German-to-English task. When compared to other typical NMT architectures, our trained ConvS2S performs significantly better than RNNbased NMT and achieves performance comparable to Transformer.

\section{CONVS2S MACHINE TRANSLATION}

Convolutional neural networks (CNNs) have several advantages over RNNs, such as fixed-size local features and high parallelization efficiency, but they have only recently been introduced into sequence modeling with diverse $\mathrm{CNN}$ implementations $[4,11,14]$. None of these implementations demonstrated improvement over state-of-the-art RNNs on large-scale datasets until the emergence of Fairseq convolutional architectures [6,7], among which ConvS2S is the winning architecture that is fully based on CNNs [7].

Both the encoder and decoder of ConvS2S are stacked deep CNN layers. Figure 1 illustrates the overall network of ConvS2S. On the source side, convolution is applied to encode local features on source word representations that the $\mathrm{RNN}$ is not able to capture, and as convolution layers are stacked deeper, wider range local features can be modeled on the source side. On the target side, deep CNN layers are also stacked that carry wide-range history features of previously generated target words. The connection between the source side and the target side is modeled by the attention layers between the last encoder CNN layer and all decoder CNN layers. Finally, a probability distribution over all translation word candidates is computed based on the current translation state summarized by the last attention layer and the output layer.

In detail, both sides of ConvS2S can be separated into two parts: word representations and deep CNN layers. The word representations part consists of word embeddings that are usually adopted in NMT networks and position embeddings that are introduced into ConvS2S to consider sequentially ordering information complementary to the convolution computation. The deep CNN layers extracts wide range local features over the word representations. Because deep layers often cause the gradient vanishing and gradient explosion problems [2], residual 


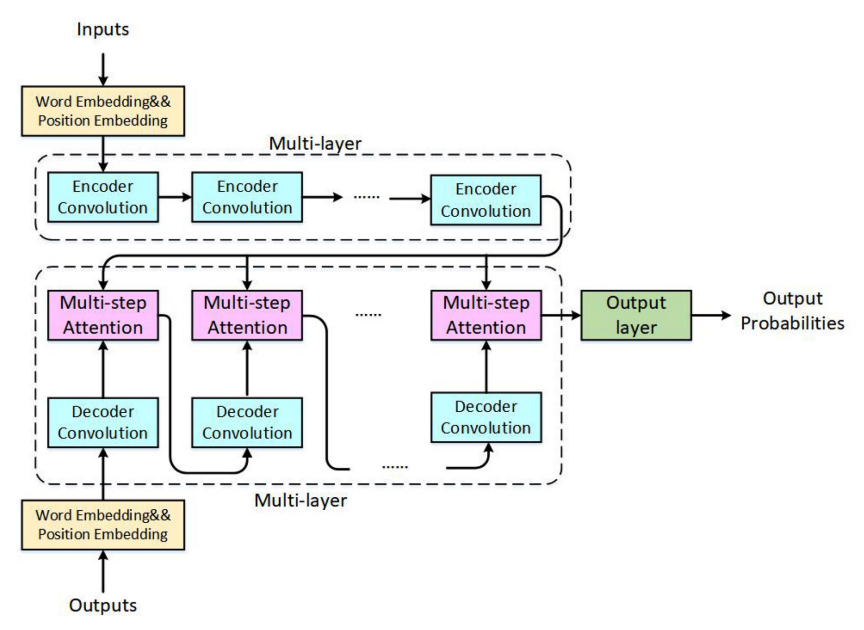

Fig. 1. Network configuration of ConvS2S.

connection [8] is applied to alleviate the two problems:

$$
h_{i}^{l}=h_{i}^{l-1}+\Theta\left(W^{l}\left(h_{i-k / 2}^{l-1}+\cdots+h_{i+k / 2}^{l-1}\right)+b^{l}\right),
$$

where $h_{i}^{l}$ denotes the hidden state of layer $l$ for the $i$-th position, $k$ denotes the convolution kernel size, $\Theta$ denotes the activation function of gated linear units [7], and $W^{l}$ denotes the convolution filter of layer $l$. The convolution outcome (e.g., $\Theta$ function output) is added to $h_{i}^{l-1}$ by the residual connection to provide a direct error propagation channel from the last layer's hidden state.

The connection between both sides of ConvS2S is modeled by the multi-step attention. Different from the traditional attention mechanism that is based on hidden states of both sides, attention weight $a_{i, j}^{l}$ in the multi-step attention is computed as follows:

$$
\begin{aligned}
a_{i, j}^{l} & =\operatorname{att}\left(z_{j}^{L}, s_{i}^{l}\right), \\
s_{i}^{l} & =W^{l} \vec{s}_{i}^{l}+b^{l}+e_{i}^{t},
\end{aligned}
$$

where att denotes the dot attention function that is based on both $z_{j}^{L}$, which is the encoder final layer output, and $s_{i}^{l}$, which is a transformation of the hidden vector $\widetilde{s}_{i}$ of the $l$-th decoder layer. Here, $b^{l}$ denotes the bias vector and $e_{i}^{t}$ is the $i$-th target word embedding.

Based on the attention weights, the context vector $c_{i}^{l}$ summarizes the source side as follows:

$$
c_{i}^{l}=\sum_{j=1}^{n} a_{i, j}^{l}\left(z_{j}^{L}+e_{j}^{s}\right) .
$$

Note that the summary is the encoder final layer output $z_{j}^{L}$ and the $j$-th source word embedding $e_{j}^{s}$. Once the context vector $c_{i}^{l}$ is obtained, it is fed to the next decoder layer together with the output of the decoder convolution $\vec{s}_{i}^{l}$ as illustrated in Figure 1.

\section{LAYER-WISE DE-TRAINING AND RE-TRAINING FOR CONVS2S}

ConvS2S is difficult to train because it often has deeper layers than RNN-based architecture or Transformer, and the trained large volumes of parameters constitute a local optimum that is hard to escape. We tried several methods in our pre-studies to avoid sticking to the local optimum. The 


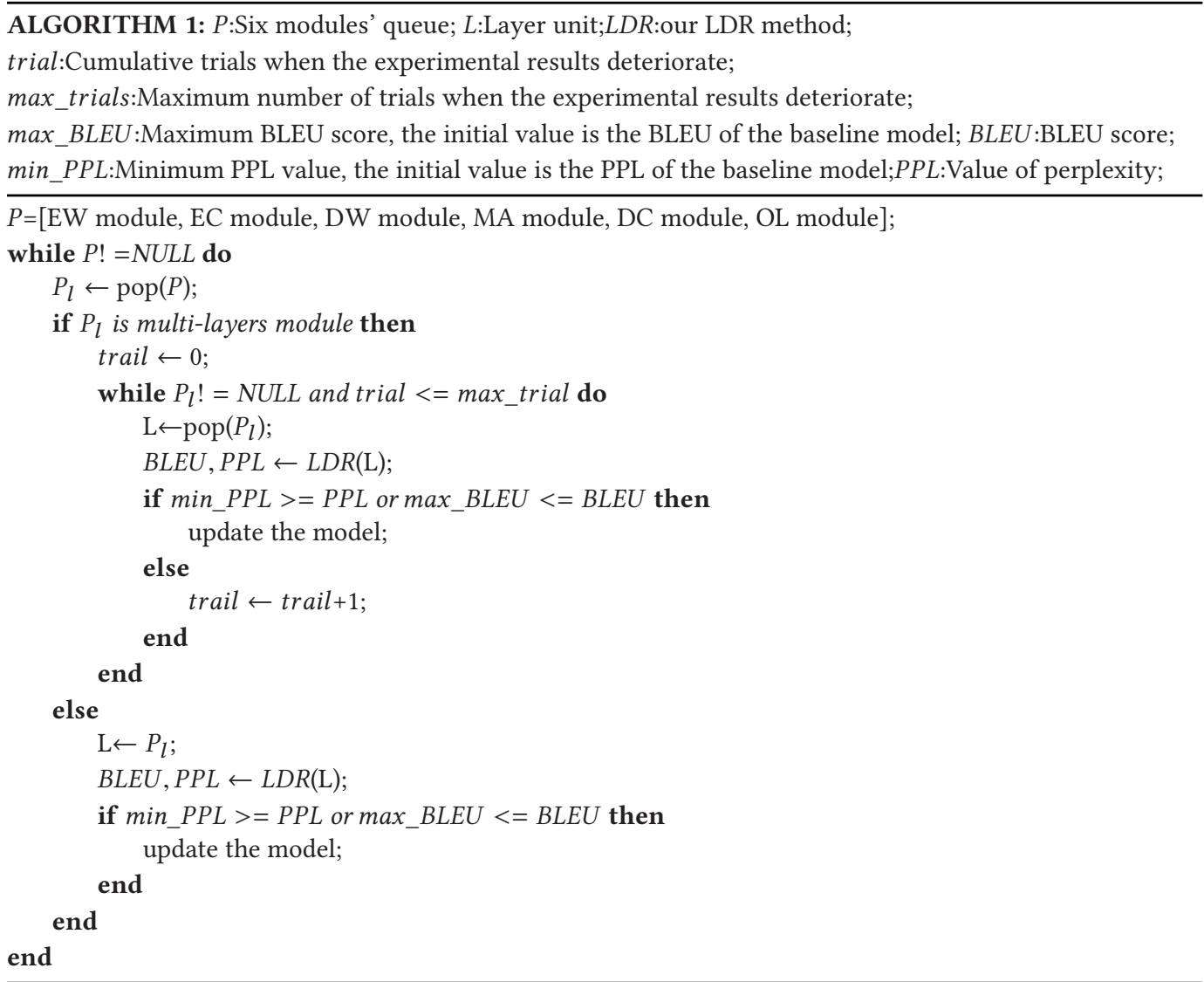

methods include initialization with random seeds, adding perturbations to the trained parameters, and training with a smaller learning rate [5]. We find that they are not able to obtain better parameter solutions than the baseline. The detailed comparisons are presented in Section 4.4.

Instead, we propose to gradually escape from the local optimum by the layer-wise de-training and re-training (LDR) procedure. In the de-training step, the local optimum is partially erased by re-initializing a specific layer and other layers remaining the same. In the re-training step, layer units are trained to find a better solution globally. In LDR, the preceding two steps are repeated layer wisely until the entire network is traversed.

\subsection{Grouped Layers of ConvS2S}

The previous section shows that our LDR method needs to be traversed in layers. To distinguish the different layer units, we segment the overall network into multi-layer modules and independent modules. As schematically illustrated in Figure 1, multi-layer modules consist of the encoder convolution (EC) module, the decoder convolution (DC) module, and the multi-step attention (MA) module. The remaining parts are considered as independent modules, consisting of the encoder word representations (EW) module, the decoder word representation (DW) module, and the output layer $(\mathrm{OL})$ module. In particular, these independent modules are regarded as three separate layer units in our experiments. 


\subsection{LDR Algorithm}

We give a detailed introduction about the two steps in the proposed scheme. The de-training step is equivalent to abandoning the one layer of the deep CNN layers. Specifically, the parameters of one layer are initialized with the initialization parameters when the CNN model is constructed, and the other parameters remain unchanged. Based on this, we re-train the combined network in the re-training step. It is worth noting that the hyper-parameter settings of the re-training step are consistent with that of training the baseline model.

The LDR algorithm is summarized in Algorithm 1. Six modules of the entire network are contained in $P$, and each module is experimented in turn. For multi-later modules, only one layer unit pops up $L$ at a time. After that, we apply the LDR method on this layer unit, and then achieve the BLEU score and the perplexity of the new model. When BLEU is greater than or equal to max_BLEU or PPL is less than or equal to min_PPL, we update the model and continue to experiment on this basis. For independent modules, we consider them as separate layer units for experimentation. Due to the excessive number of layer units in the multi-layer module, we have added the constraint trial. When the experimental results are not good, we accumulate the number of the trial. Once the value of trial exceeds max_trial, the entire module is discarded and we proceed to the next module. Through the LDR algorithm, all modules will be traversed until the end.

\subsection{Bidirectional LDR}

Rather than blindly traversing the modules, two brief traversal strategies are adopted: the forward strategy and the backward strategy. The sequence of the forward strategy is in accordance with $\mathrm{EW}$ module $\rightarrow \mathrm{DW}$ module $\rightarrow \mathrm{EC}$ module $\rightarrow \mathrm{MA}$ module $\rightarrow \mathrm{DC}$ module $\rightarrow \mathrm{OL}$ module. In each multilayer module, we also carried out experiments in accordance with the layer sequence from front to back. For example, the experimental sequence of a 16-layer CNN is from the 1st layer to the 16th layer. Analogously, the backward strategy is the reverse of the the forward strategy, including the layer units.

The ensemble method [9] has been widely used in the field of machine translation, and our commonly utilized ensemble method is to average the prediction results of each word by considering the results of multiple models during decoding. The combination of multiple models can improve the generalization ability of the entire network. Therefore, we ensemble the two models of the forward strategy and the backward strategy in the experiment, and further improve the experimental results.

\section{EXPERIMENTS}

\subsection{Data}

We evaluate our experimental methods on the widely used NIST Chinese-English translation task. To enhance the reliability of our experimental method, we also conduct an experiment on the WMT17 German-English translation task.

For the Chinese-English data, the training set is extracted from the Linguistic Data Consortium (LDC) corpora, including 1.25 million sentence pairs. ${ }^{1}$ The test sets use the National Institute of Standards and Technology data NIST02, NIST03, NIST04, NIST05, and NIST08, where NIST06 is the development set. The vocabulary size is limited to 30,000 , and all of the out-of-vocabulary words with low frequency are mapped to the special token $<U N K>$.

\footnotetext{
${ }^{1}$ The corpora includes LDC2002E18, LDC2003E07, LDC2003E14, Hansards portion of LDC2004T07, LDC2004T08, and LDC2005T06.
} 
For the German-English data, we use the WMT17 [3] corpus as our training data. The newstest2016 is used as the development set and newstest2017 as the test set. There are about 5.5 million sentence pairs in the training set after preprocessing with Moses script and filtering the sentences longer than 50. In addition, we applied the byte pair encoding algorithm [18] to encode all GermanEnglish sentences and limited the vocabulary size to 90,000.

We evaluate translation quality via BLEU scores [15] and use the multi-bleu script. For ChineseEnglish data, we apply the case-insensitive 4-gram BLEU score as the basis for evaluation. For German-English data, we use the case-sensitive and case-insensitive BLEU score as the evaluation standard. In addition, during decoding, we use the beam search algorithm, and the beam search is set to 10 .

\subsection{Experimental Setting}

We apply the LDR method to the ConvS2S model and fine tune the model structure on different datasets.

For the Chinese-English model, we stack 16-layer CNNs at both encoders and decoders. For each convolution layer, the kernel size is set to 3 , and all dimensionalities of the hidden units and embeddings are set to $256,{ }^{2}$ including the size of each linear layer.

In addition, the ConvS2S model employs the optimizer with Nesterov's accelerated gradient method [20], which uses a momentum value of 0.99 and renormalizes gradients if the norm exceeds 0.1 [16]. The learning rate is set to 0.25 , and the training ends when it drops below $1 \mathrm{e}-5$. In training, the learning rate will be decayed as long as the validation loss plateaus. All of the models are implemented in PyTorch [17] and trained with one GPU. During training, each mini-batch contains 6,000 tokens, and dropout [19], which is applied to the whole network, is set to 0.2 .

For the German-English model, it is basically consistent with the configuration of the Chinese and English models, but there are some differences in the hyperparameter settings. We increased the number of convolution layers at both ends to 20 layers. Each mini-batch contains 3,000 tokens, and the dropout is changed to 0.1 . As the amount of data increases, we use two GPUs during training.

\subsection{Experimental Results}

Table 1 mainly shows the translation performance of ConvS2S, RNN, Transformer, and indicates the results of the LDR method including forward and Backward strategies on Chinese-English and German-English data.

Comparing the other systems, the ConvS2S achieves good translation performance. For RNNS2S, we use a single-layer bidirectional LSTM with a default parameter on the Chinese-English and German-English tasks. In addition, Transformer achieves the highest BLEU score on both tasks. Nevertheless, for German-English data, Transformer, whose results are consistent with other studies [23], does not achieve good experimental results when using the single GPU; we readjust parameters and the number of GPUs.

From the perspective of BLEU scores, it is obvious that our two strategies achieve quite good experimental results. On all test sets, the experimental results outperform the baseline ConvS2S by 2.964 BLEU points on the Chinese-English task and 0.94 BLEU points on the German-English task. Through the ensemble method, two strategies are combined to further improve the results, which outperform 3.688 BLEU points on the Chinese-English task and 1.04 BLEU points on the German-English task.

\footnotetext{
${ }^{2}$ We also tried the 512-dimensional model. The BLEU value of Chinese and English is 38, and the BLEU value of German and English is 29.98 .
} 
Table 1. Experimental Results of Different Strategies on the Chinese-English and German-English Translation Tasks

\begin{tabular}{|c|c|c|c|c|c|c|c|}
\hline Corpus & Model & Nist02 & Nist03 & Nist04 & Nist05 & Nist08 & Average \\
\hline \multirow{6}{*}{$\mathrm{CH}-\mathrm{EN}$} & ConvS2S* & 40.43 & 38.14 & 40.8 & 38.5 & 29.29 & 37.432 \\
\hline & RNNS2S & 39.59 & 36.66 & 40.05 & 37.31 & 28.7 & 36.462 \\
\hline & Transformer & 42.56 & 41.12 & 43.98 & 41.71 & 32.07 & 40.288 \\
\hline & Forward strategy & 42.53 & 41.54 & 43.23 & 41.03 & 31.77 & 40.02 \\
\hline & Backward strategy $^{\dagger}$ & 43.65 & 40.83 & 44.04 & 41.72 & 31.74 & 40.396 \\
\hline & Ensemble & 44.08 & 41.89 & 44.5 & 42.45 & 32.68 & 41.12 \\
\hline Corpus & Model & \multicolumn{3}{|c|}{ Case Sensitive } & \multicolumn{3}{|c|}{ Case Insensitive } \\
\hline \multirow{6}{*}{ GE-EN } & ConvS2S* & \multicolumn{3}{|c|}{29.51} & \multicolumn{3}{|c|}{30.72} \\
\hline & RNNS2S & \multicolumn{3}{|c|}{28.2} & \multicolumn{3}{|c|}{29.39} \\
\hline & Transformer & \multicolumn{3}{|c|}{31.95} & \multicolumn{3}{|c|}{34.75} \\
\hline & Forward strategy $^{\dagger}$ & \multicolumn{3}{|c|}{30.1} & \multicolumn{3}{|c|}{31.3} \\
\hline & Backward strategy $^{\dagger}$ & \multicolumn{3}{|c|}{30.41} & \multicolumn{3}{|c|}{31.66} \\
\hline & Ensemble & \multicolumn{3}{|c|}{30.54} & \multicolumn{3}{|c|}{31.76} \\
\hline
\end{tabular}

The asterisk $\left(^{*}\right)$ represents our baseline system. The dagger $(\dagger)$ represents our experimental strategies.

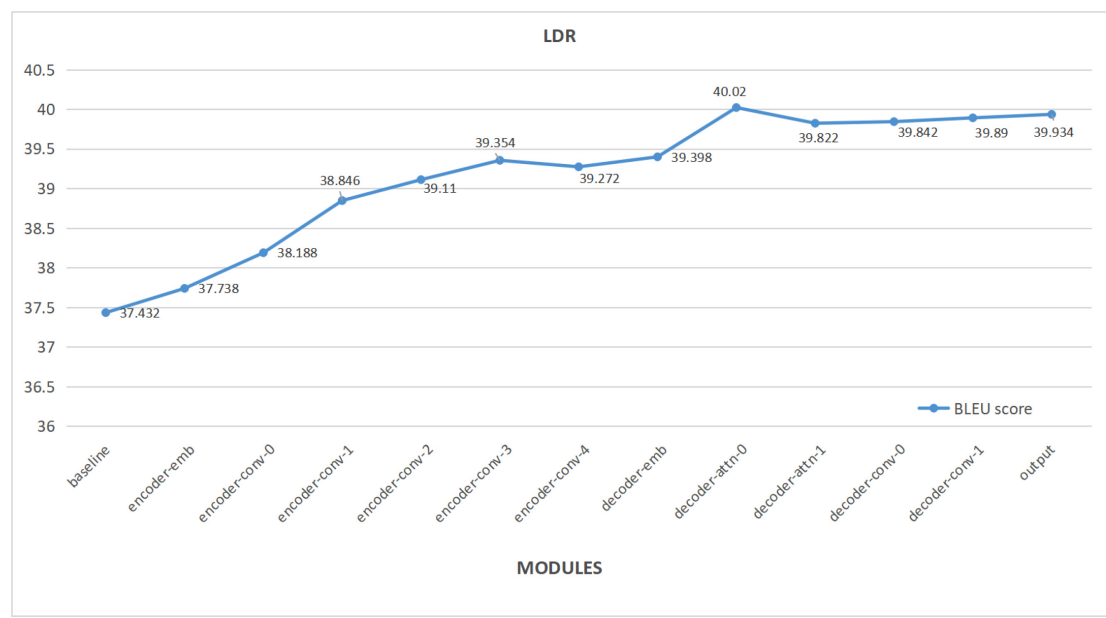

Fig. 2. The specific experimental results of the Chinese-English forward strategy. The abscissa is the order of the initialization weights, and the ordinate indicates the Chinese-English BLEU scores and valid loss.

As illustrated in Figure 2, we show specific experimental procedures of the forward strategy. In the Chinese-English task, we conducted four layers of experiments on the EW module, two layers on the DA modules, and two layers on the DC modules. In addition, the EW module, the DW module, and the OL module are traversed once. Specifically, the BLEU scores of each experiment are shown in the figure, and we can clearly see that the BLEU scores rise rapidly.

\subsection{Comparison to Tentative Methods of Escaping from Local Maximum}

To escape from the local optimum, we also attempted three methods to deal with such problems. The first way is to attempt to change the random number seed. The fixed random number seed is removed in the model to keep the initialization value of each parameter more random. The second method is to attempt to add perturbations to the parameters of the baseline model. The 
Table 2. Results of Different Models and Three Tentative Methods on Chinese-English and German-English Data

\begin{tabular}{|c|c|c|c|c|c|c|c|}
\hline \multicolumn{2}{|c|}{ LDC Chinese-English } & Nist02 & Nist03 & Nist04 & Nist05 & Nist08 & Average \\
\hline \multirow{3}{*}{ Random seeds } & randn_seed 1 & 40.86 & 38.34 & 40.54 & 38.86 & 29.11 & 37.542 \\
\hline & randn_seed 2 & 40.57 & 37.81 & 40.49 & 37.8 & 28.92 & 37.118 \\
\hline & randn_seed 3 & 40.21 & 38.37 & 40.9 & 38.09 & 29.38 & 37.39 \\
\hline \multirow{2}{*}{ Add perturbations } & best epoch $(\mathrm{std}=0.001)$ & 40.67 & 38.28 & 40.74 & 38.46 & 29.3 & 37.49 \\
\hline & best epoch $(\mathrm{std}=0.005)$ & 39.92 & 38.25 & 40.66 & 38.23 & 29.25 & 37.262 \\
\hline \multirow{3}{*}{ Con-training } & 10th epoch & 40.5 & 38.32 & 40.77 & 38.55 & 29.16 & 37.46 \\
\hline & 20th epoch & 40.55 & 38.22 & 40.81 & 38.49 & 29.17 & 37.448 \\
\hline & 30th epoch & 40.55 & 38.22 & 40.8 & 38.51 & 29.2 & 37.456 \\
\hline \multicolumn{2}{|c|}{ WMT17 German-English } & \multicolumn{3}{|c|}{ Case Sensitive } & \multicolumn{3}{|c|}{ Case Insensitive } \\
\hline \multirow{3}{*}{ Random seeds } & randn_seed 1 & \multicolumn{3}{|c|}{28.41} & \multicolumn{3}{|c|}{29.59} \\
\hline & randn_seed 2 & \multicolumn{3}{|c|}{28.1} & \multicolumn{3}{|c|}{29.26} \\
\hline & randn_seed 3 & \multicolumn{3}{|c|}{28.35} & \multicolumn{3}{|c|}{29.59} \\
\hline \multirow{2}{*}{ Add perturbations } & best epoch $(\mathrm{std}=0.001)$ & \multirow{2}{*}{\multicolumn{3}{|c|}{$\begin{array}{l}29.28 \\
29.37\end{array}$}} & \multicolumn{3}{|c|}{30.51} \\
\hline & best epoch $(\mathrm{std}=0.005)$ & & & & \multicolumn{3}{|c|}{30.6} \\
\hline \multirow{3}{*}{ Con-training } & 10th epoch & \multicolumn{3}{|c|}{29.44} & \multicolumn{3}{|c|}{30.67} \\
\hline & 20th epoch & \multicolumn{3}{|c|}{29.43} & \multicolumn{3}{|c|}{30.66} \\
\hline & 30th epoch & \multicolumn{3}{|c|}{29.41} & \multicolumn{3}{|c|}{30.63} \\
\hline
\end{tabular}

Results are reported with multi-bleu.perl. The case-insensitive 4-gram BLEU is applied as the Chinese-English evaluation standard. The case-sensitive and case-insensitive BLEU is applied as the German-English evaluation standard.

perturbations, whose weights are initialized from a normal distribution with a mean of 0 and a standard deviation of 0.001 or 0.005 , are added correspondingly to the parameters of different positions. The combined parameters are continued to train with the smaller learning rate. The third method is to continue training with the baseline model with a smaller learning rate. Specifically, after loading the baseline model, we made attempts to continue training the model with the smaller learning rate and update the model parameters more carefully with smaller steps.

The data in Table 2 indicate the results of our attempt on three approaches of random seed, adding perturbations, and con-training. For the first approach, after canceling the fixed random number seed, the effect of training for the entire network is still fluctuating around the result of the baseline. For the second method of adding perturbations, we use two categories of perturbations. For perturbations with a standard deviation of 0.001 and 0.005 , we selected the best epoch in the training process, and the results were basically unchanged. Meanwhile, we found that the value of its verification set loss was almost unchanged during the training. We also tried a larger standard deviation, but it causes training confusion. In the third method, we attempt to continue training the baseline model, but it still does not work.

Summarizing the preceding three results, whether on the Chinese-English or the GermanEnglish task, the most straightforward judgment is that the three methods we attempted have no significant changes in the BLEU scores. Otherwise, it can be inferred that these three methods cannot effectively address the local optimum problem.

\subsection{Comparison with RNNS2S and Transformer}

We also apply our approach to other models, such as RNNS2S and Transformer. As illustrated in Table 3, LDR is also able to improve the performance of both RNNS2S and Transformer, but the improvement is marginal compared to the significant improvements of using LDR on ConvS2S. We 
Table 3. Experimental Results of LDR Applied to RNN and Transformer

\begin{tabular}{l|c|ccccc|c}
\hline Corpus & Model & Nist02 & Nist03 & Nist04 & Nist05 & Nist08 & Average \\
\hline \multirow{4}{*}{ CH-EN } & RNNS2S & 39.59 & 36.66 & 40.05 & 37.31 & 28.7 & 36.462 \\
& RNNS2S+LDR & 40.26 & 38.44 & 40.97 & 38.46 & 29.71 & 37.568 \\
& Transformer & 42.56 & 41.12 & 43.98 & 41.71 & 32.07 & 40.288 \\
& Transformer+LDR & 43.59 & 41.98 & 43.98 & 41.88 & 32.62 & 40.81 \\
\hline \hline Corpus & Model & Case Sensitive & \multicolumn{3}{c}{ Case Insensitive } \\
\hline \multirow{3}{*}{ GE-EN } & RNNS2S & \multicolumn{3}{c}{28.2} & & 29.39 & 29.69 \\
& RNNS2S+LDR & & 28.47 & & 34.75 & \\
& Transformer & & 31.95 & & 35.04 & \\
& Transformer+LDR & 32.26 & & & & & \\
\hline
\end{tabular}

Table 4. Experimental Results on Different Parameters

\begin{tabular}{l|ccccc|c}
\hline Model & Nist02 & Nist03 & Nist04 & Nist05 & Nist08 & Average \\
\hline ConvS2S(adam) & 41.02 & 38.56 & 41.35 & 39.63 & 31.15 & 38.342 \\
ConvS2S(adam)+LDR & 43.53 & 41.7 & 44.54 & 42.54 & 33.07 & 41.076 \\
ConvS2S(512dim, multi-GPU) & 41.1 & 38.91 & 41.44 & 39.21 & 29.47 & 38.026 \\
ConvS2S(512dim, multi-GPU)+LDR & 42.23 & 40.88 & 42.84 & 40.83 & 30.89 & 39.534 \\
\hline
\end{tabular}

are still studying the specific reasons for the poor performance of the two models. We consider that it may be that RNNS2S and Transformer are relatively complete for extracting the relationship information between words and words. As a neural network of time series, RNNS2S easily allows the sentence information to transmit backward in a constant manner. Due to its self-attention mechanism, the Transformer model is more explicit about the interrelationship between words.

\subsection{Comparison with Different Parameters}

In Table 4, we compared the effects of different parameters on the Chinese and English datasets of the LDR method. First, we chose adam as the optimizer and set the learning rate at 0.0005 . Moreover, during the experiment, we found that using the adam optimizer can find the better optimum faster and achieve better experimental results. In addition, we experimented with the baseline of 512-dimension embedding and hidden states using multi-GPU; the results show that our previous baseline in Table 1 performs comparable to this new baseline under the framework of ConvS2S. Furthermore, we can further improve the performance of the new baseline by using our LDR method, as shown in the last row of Table 4.

\section{ANALYSIS}

The main reason for CNNs to generate the local optimum is that the excessive stacked deeper layers are difficult to train completely. In other words, not all parameters of the layer have reached an optimal value after training. For the whole network, all parameters are interrelated in the process of forward propagation. Changes in a certain layer of the network will directly affect the subsequent parameters. Specifically, we can change the value of one layer in the module by the de-training step so as to affect the whole network. Based on this, we re-adjust the parameters of the network by the re-training step. The two steps are then iteratively repeated, constantly improving the translation model and making the neural network stronger and more robust. According to our algorithm, the multi-layer network structure will be traversed to progressively reach a state that tends to be globally optimal. 


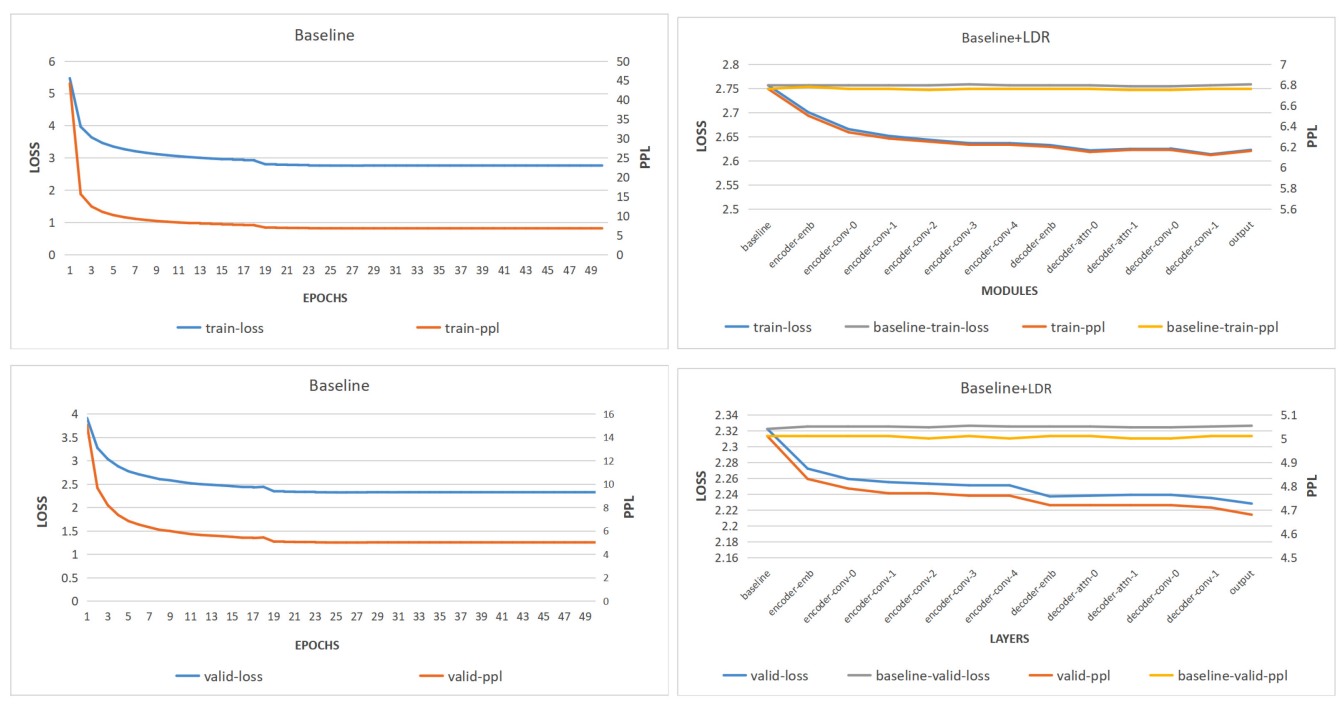

Fig. 3. Comparison of loss and ppl in the training set and the development set.

\subsection{Analysis of Evaluation Indicators}

We validate the effectiveness of our approach by evaluating indicators. The left-hand sides in Figure 3 show the performance of our baseline model on German and English data. The 27th epoch previously was the training process of the baseline model, followed by the experimental results using the con-training method. From the figure, we can clearly see that in the training process, the loss and PPL on the development set are not an obvious downward trend, which proves that the model parameters have reached the "optimal state." Nevertheless, it does not mean that the model does achieve the "global optimum," and we believe that it is stuck in the local optimum. The right-hand sides in Figure 3, comparing the indicators obtained by the baseline model using the con-training method with the indicators after using the LDR method, it can be clearly seen that both the loss and ppl of the training set and development set can be further reduced. The curves of loss and ppl are overlapped since the trend of the declines is similar. But this still shows that the performance of the model is constantly improving. Specifically, the downward trend in loss and ppl will be more prominent after using the LDR method for EW, DW, and OL modules. The specific analysis will be analyzed in detail in Section 5.3.

The LDR method that we proposed is similar to a kind of hill-climbing method. Nevertheless, we have been using the valid set to monitor whether the data of the training set will over-fitting during the training process. It can be found from Figure 3 that the loss and ppl are both falling normally, and the downward trend is basically similar. The situation where the decrease of ppl in the training data is accompanied by the increase in development set do not appear.

\subsection{Principal Component Analysis}

When analyzing the model parameters, we employ the principal component analysis (PCA) $[10,12]$ method, which is a commonly used data-mapping method in machine learning. The PCA method mainly finds the eigenvectors corresponding to the largest eigenvalues of the covariance matrix of the dataset, thereby finding the directions with the largest data variance, reducing the dimensionality of the data, and reducing an $n$-dimensional vector to a $d$-dimension, wherein $d<n$. 


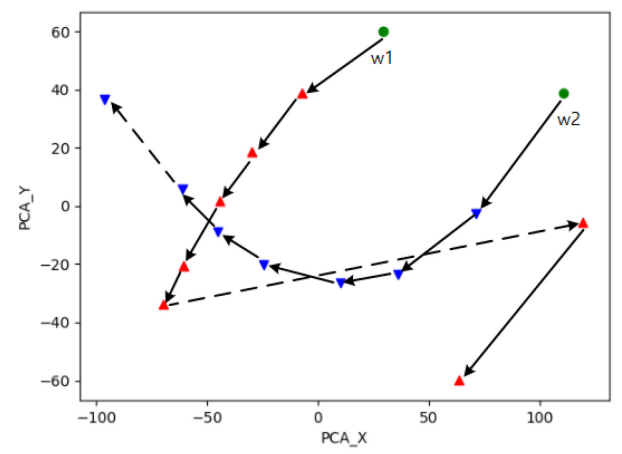

Fig. 4. Two examples of weight change processes. We selected seven models traversed in the forward strategy process, and each model was selected with two weights as examples. "w1" and "w2" represent the weights of the output layer and the decoder word embedding, respectively. " $\bigcirc$ " (green) indicates the weight of the baseline model. " $\nabla$ " (blue) and " $\Delta$ " (red) represent changes of two weights. The solid arrow represents changes that do not utilize the LDR method. The dotted arrow represents changes utilizing the LDR method.
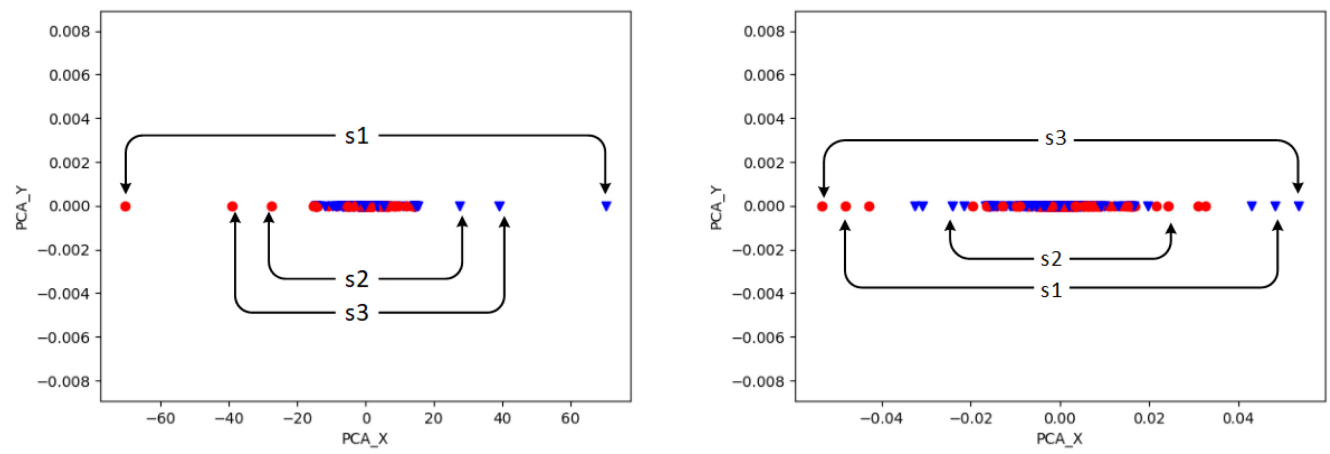

Fig. 5. Comparison of the scatter plot of all weights between the baseline model and the other model. " $\bigcirc$ " (red) represents the weights of the baseline model. Left: Comparison between the baseline model and the best model of the LDR forward strategy. " $\nabla$ " (blue) represents the weights of the LDR model. Right: Comparison between the baseline model and the best model of con-training method. " $\nabla$ " (blue) represents the weights of the con-training model. "s1," "s2," and "s3" represent three large-dimensional vectors of the encoder word embedding, the decoder word embedding, and the output layer, respectively.

In Figure 4, we reduce the two weights of the seven models in the forward strategy process to two-dimensional coordinates by PCA. "w1" and "w2" represent the weights of the output layer and the decoder word embedding, respectively. The solid arrows indicate weight changes caused by other layer units using the LDR method. We can see that the two weights are adjusted continuously each time to find a more suitable location for the entire network during the traversal of the forward strategy. The dotted arrows indicate the change in weight after using the LDR method. Obviously, both weights have changed dramatically after using the LDR method, especially the "w1" weight. In each traversal, the weights can jump out of the local optimum and find a better state that tends to be a global optimum state gradually. In addition, we also observed the change of the gradient of the parameters and found that it remained in a state of change during the training.

Furthermore, we will compare all of the weights between the LDR method and the con-training method in Figure 5. In the left figure, the baseline model is compared to the best model obtained by the LDR method. The figure on the right is the comparison between the baseline model and 
Table 5. Data Comparison of Model Weights from Three Aspects, Which Consist of Euclidean Distance, Mean, and Variance

\begin{tabular}{c|c|cc}
\hline Weights & Measure & Baseline & LDR \\
\hline \hline \multirow{3}{*}{ Encoder-emb } & Distance & \multicolumn{2}{|c}{141.24} \\
& Mean & $1.78^{*} 1 \mathrm{e}-05$ & $1.351 \mathrm{e}-05$ \\
& Std & $1.39^{*} 1 \mathrm{e}-01$ & $1.21^{*} 1 \mathrm{e}-01$ \\
\hline \multirow{3}{*}{ Decoder-emb } & Distance & \multicolumn{2}{|c}{54.95} \\
& Mean & $5.32^{*} 1 \mathrm{e}-05$ & $5.72^{*} 1 \mathrm{e}-05$ \\
& Std & $1.17^{*} 1 \mathrm{e}-01$ & $1.22^{*} 1 \mathrm{e}-01$ \\
\hline \multirow{3}{*}{ Output-weight } & Distance & \multicolumn{2}{|c}{78.28} \\
& Mean & $5.86^{*} 1 \mathrm{e}-04$ & $6.87^{*} 1 \mathrm{e}-04$ \\
& Std & $1.06^{*} 1 \mathrm{e}-01$ & $1.17^{*} 1 \mathrm{e}-01$ \\
\hline \hline \multirow{3}{*}{ Weights } & Measure & Baseline & Con-training \\
\hline \hline & Distance & \multicolumn{2}{|c}{$8.60^{*} 1 \mathrm{e}-02$} \\
& Mean & $1.78^{*} 1 \mathrm{e}-05$ & $1.781 \mathrm{e}-05$ \\
& Std & $1.39^{*} 1 \mathrm{e}-01$ & $1.39^{*} 1 \mathrm{e}-01$ \\
\hline \multirow{3}{*}{ Decoder-emb } & Distance & \multicolumn{2}{|c}{$4.85^{*} 1 \mathrm{e}-02$} \\
& Mean & $5.32^{*} 1 \mathrm{e}-05$ & $5.32^{*} 1 \mathrm{e}-05$ \\
& Std & $1.17^{*} 1 \mathrm{e}-01$ & $1.17^{*} 1 \mathrm{e}-01$ \\
\hline \multirow{3}{*}{ Output-weight } & Distance & \multicolumn{2}{|c}{$9.62^{*} 1 \mathrm{e}-02$} \\
& Mean & $5.86^{*} 1 \mathrm{e}-04$ & $5.84^{*} 1 \mathrm{e}-04$ \\
& Std & $1.06^{*} 1 \mathrm{e}-01$ & $1.06^{*} 1 \mathrm{e}-01$ \\
\hline
\end{tabular}

the 30th model obtained by the con-training method. The red and blue scatter points represent the baseline model parameters and the parameters of the other model, respectively. In addition, it can be seen that the two figures are basically symmetrical, because all model parameters are compared in pairs so that we can directly see the difference between the two weights. Nevertheless, it is easily confused because of too many model parameters. So we have selected a few distinct points, s1, s2, and s3, which represent the encoder word embedding, the decoder word embedding, and the output layer, respectively. The commonality of these weights is that they are all large-dimensional vectors in the model parameters, whose variation of the values in the experiment are also relatively obvious. Moreover, we can still compare the coordinates of the two graphs to conclude that the changes between the baseline model and model obtained by our LDR are huge. For more detailed judgment of weight changes, we will analyze the weights more specifically in the next section.

\subsection{Weight Analysis}

In Table 5, three large-dimensional vectors, which consist of the encoder word embedding, the decoder word embedding, and the output layer, are analyzed in detail. Similarly, in the upper part of the table is the comparison between the baseline model and the best model obtained by LDR, and the lower half is also the comparison between the baseline model and the 30th model obtained by the con-training method. We measure the change between weights from three perspectives that consist of Euclidean distance, mean, and standard deviation. By comparing the Euclidean distances of the two tables, it is obvious that the best we achieve is far from the local optimum of the baseline. From the comparison of the mean and the standard deviation, we can also find that the numerical changes of the con-training model and the baseline model are very small-almost zero. 
Table 6. Comparison of Resources Required for Training

\begin{tabular}{c|c|c|c}
\hline Chinese-English & Baseline $(\mathrm{CNN})$ & Baseline+LDR & Transformer \\
\hline \hline Total training time & $11 \mathrm{~h}$ & $64 \mathrm{~h}$ & $26 \mathrm{~h}$ \\
Memory consumption & $1.3 \mathrm{~K}$ & $1.3 \mathrm{~K}$ & $2.6 \mathrm{~K}$ \\
\hline \hline
\end{tabular}

Table 7. Examples of Translations on the German-English of the newtest2017 Dataset at Different Traversal Times of the LDR Backward Strategy

\begin{tabular}{|c|c|}
\hline Source & Behaupteten beide Männer, dass der Stabschef auf eigenen Wunsch zurücktritt. \\
\hline Baseline & Both men claimed that the Chief of Staff returned to his own desire. \\
\hline 1st traversal & Both men claimed that the Chief of Staff resigned at his own request. \\
\hline 8th traversal & Both men claimed that the chief of staff has resigned at their own request. \\
\hline Best traversal & Both men claimed that the chief of staff would resign at his own request. \\
\hline Reference & Both men claimed that the chief of staff was stepping down at his own request. \\
\hline Source & $\begin{array}{l}\text { Jetzt ist der 30-Jährige selbst bei einem Fassbomben-Angriff des Assad-Regimes } \\
\text { gestorben. }\end{array}$ \\
\hline Baseline & Now the 30-year-old has died from the Assad regime's Fassbomben-attack. \\
\hline 1st traversal & Now the 30-year-old has died from the Assad regime's Fassbomben-attack. \\
\hline 8th traversal & Now the 30-year-old has died from the Assad regime's bombing attack. \\
\hline Best traversal & Now, the 30 year old has died even with a barrel bomber attack by the Assad regime. \\
\hline Reference & Now the 30 -year-old has been killed in a barrel bomb attack by the Assad regime. \\
\hline
\end{tabular}

We show translations at First, Eighth and best traversal, where the model obtains case-sensitive BLEU scores of 29.83, 30.32 and 30.41 respectively.

Furthermore, as illustrated in Figures 3 and 4, we have observed that three modules (i.e., EW, DW, and OL) experience significant changes, which lead to better optimization of the model. The reason these modules have a great influence on the network is that the three modules contain largedimensional matrices that take up a large portion of the parameters of the neural network. After the LDR method is applied, the encoder word embedding and decoder word embedding effectively extract the information from the sentences. When we apply the LDR method to the output layer, it can be combined with the information of the previous network stream to redirect the information at the output, resulting in a better probability distribution.

\subsection{Resource Analysis}

The LDR method is similar to a progressive hill-climbing method. The obvious disadvantage is that the training time will be relatively long. As shown in Table 6, for the Chinese and English data, all of our training time is 64 hours, which is approximately six times longer than the baseline system, and about 2.5 times longer than the transformer system. On the other hand, the memory consumption of ConvS2S is relatively small, only about half that of Transformer. When comparing the memory consumption, we also unified the value of max_tokens and consider only the memory occupied by the model parameters, which is basically half the memory consumption.

\subsection{Qualitative Analysis}

Table 7 shows the German-English translation of English sentences in the newtest2017 dataset at different traversal times of the LDR backward strategy. As we increase number of traversals, we observe continuous improvement in translation quality. In the first example, the tense of the German word zurücktritt, which can be translated as "resign" or "step down," is constantly changing 
during the traversal. More importantly, when the baseline model was translated into "return," the word immediately is changed to "resign" after the first traversal. This is because in the first traversal, we prioritize the traversal of the output layer under the premise of using the backtracking strategy. The output layer will re-adjust its probability distribution after using the LDR method, and it makes the output layer more suitable for the entire network.

\section{CONCLUSION}

In this experiment, we propose a simple and novel method to address the local optimum problem. Considering the relationship between the whole and the part and the construction of the neural network, the LDR method we propose can get rid of the local optimum. The experimental results also show that our experimental method has reached a fairly high level in the Chinese-English results, basically reaching the translation level of the transformer, and our method is equally applicable to different corpus pairs. In future work, we will further expand our methods in low-resource machine translation, especially for local best optimum.

\section{ACKNOWLEDGMENTS}

We thank for insightful comments of anonymous reviewers.

\section{REFERENCES}

[1] Dzmitry Bahdanau, Kyunghyun Cho, and Yoshua Bengio. 2015. Neural machine translation by jointly learning to align and translate. In Proceedings of the 3rd International Conference on Learning Representations.

[2] Y. Bengio, P. Simard, and P. Frasconi. 2002. Learning long-term dependencies with gradient descent is difficult.IEEE Transactions on Neural Networks 5, 2 (2002), 157-166.

[3] Ondrej Bojar, Rajen Chatterjee, Christian Federmann, Yvette Graham, Barry Haddow, Shujian Huang, Matthias Huck, Philipp Koehn, Qun Liu, and Varvara Logacheva. 2017. Findings of the 2017 conference on machine translation (WMT17). In Proceedings of the Conference on Machine Translation. 169-214.

[4] James Bradbury, Stephen Merity, Caiming Xiong, and Richard Socher. 2016. Quasi-recurrent neural networks. arXiv:1611.01576.

[5] Michael Denkowski and Graham Neubig. 2017. Stronger baselines for trustable results in neural machine translation. arXiv:1706.09733.

[6] Jonas Gehring, Michael Auli, David Grangier, and Yann N. Dauphin. 2017. A convolutional encoder model for neural machine translation. In Proceedings of the Annual Meeting of the Association for Computational Linguistics (ACL'17).

[7] Jonas Gehring, Michael Auli, David Grangier, Denis Yarats, and Yann N. Dauphin. 2017. Convolutional sequence to sequence learning. arXiv:1705.03122.

[8] Kaiming He, Xiangyu Zhang, Shaoqing Ren, and Jian Sun. 2016. Deep residual learning for image recognition. In Proceedings of the 2016 IEEE Conference on Computer Vision and Pattern Recognition (CVPR'16). 770-778.

[9] Sabastien Jean, Kyunghyun Cho, Roland Memisevic, and Yoshua Bengio. 2015. On using very large target vocabulary for neural machine translation. arXiv:1412.2007.

[10] Ian Jolliffe. 2011. Principal component analysis. In International Encyclopedia of Statistical Science. Springer, 10941096.

[11] Nal Kalchbrenner, Lasse Espeholt, Karen Simonyan, Aaron Van Den Oord, Alex Graves, and Koray Kavukcuoglu. 2016. Neural machine translation in linear time. arXiv:1610.10099.

[12] Rajiv Khanna, Joydeep Ghosh, Russell Poldrack, and Oluwasanmi Koyejo. 2017. A deflation method for structured probabilistic PCA. In Proceedings of the 2017 SIAM International Conference on Data Mining. 534-542.

[13] Minh-Thang Luong, Hieu Pham, and Christopher D. Manning. 2015. Effective approaches to attention-based neural machine translation. In Proceedings of the 2015 Conference on Empirical Methods on Natural Language Processing (EMNLP'15).

[14] Fandong Meng, Zhengdong Lu, Mingxuan Wang, Hang Li, Wenbin Jiang, and Qun Liu. 2015. Encoding source language with convolutional neural network for machine translation. In Proceedings of the Annual Meeting of the Association for Computational Linguistics (ACL'15).

[15] Kishore Papineni, Salim Roukos, Todd Ward, and Wei Jing Zhu. 2002. BLEU: A method for automatic evaluation of machine translation. In Proceedings of the Annual Meeting of the Association for Computational Linguistics (ACL'02). 311-318. 
[16] Razvan Pascanu, Tomas Mikolov, and Yoshua Bengio. 2013. On the difficulty of training recurrent neural networks. In Proceedings of the International Conference on Machine Learning. 1310-1318.

[17] Adam Paszke, Sam Gross, Soumith Chintala, Gregory Chanan, Edward Yang, Zachary DeVito, Zeming Lin, Alban Desmaison, Luca Antiga, and Adam Lerer. 2017. Automatic differentiation in PyTorch. In Proceedings of the 31st Conference on Neural Information Processing Systems (NIPS'17).

[18] Rico Sennrich, Barry Haddow, and Alexandra Birch. 2016. Neural machine translation of rare words with subword units. In Proceedings of the Annual Meeting of the Association for Computational Linguistics (ACL'16).

[19] Nitish Srivastava, Geoffrey Hinton, Alex Krizhevsky, Ilya Sutskever, and Ruslan Salakhutdinov. 2014. Dropout: A simple way to prevent neural networks from overfitting. Journal of Machine Learning Research 15, 1 (2014), 19291958.

[20] Ilya Sutskever, James Martens, George Dahl, and Geoffrey Hinton. 2013. On the importance of initialization and momentum in deep learning. In Proceedings of the International Conference on Machine Learning. 1139-1147.

[21] Ilya Sutskever, Oriol Vinyals, and Quoc V. Le. 2014. Sequence to sequence learning with neural networks. In Proceedings of the 27th International Conference on Neural Information Processing Systems (NIPS'14).

[22] Ashish Vaswani, Noam Shazeer, Niki Parmar, Jakob Uszkoreit, Llion Jones, Aidan N. Gomez, Lukasz Kaiser, and Illia Polosukhin. 2017. Attention is all you need. arXiv:1706.03762.

[23] Biao Zhang, Deyi Xiong, and Jinsong Su. 2018. Accelerating neural transformer via an average attention network. arXiv:1805.00631.

Received December 2018; revised August 2019; accepted August 2019 